\title{
BOOK REVIEWS / BOEK RESENSIES
}

\section{Juliana M. Claassens. Claiming Her Dignity. Female Resistance in the Old Testament. Collegeville: Liturgical Press, 2016. 165 pages, soft cover, price \$24.95. ISBN 978-0-8146-8419-1.}

The book starts with the sentence that "to be human means to resist dehumanization" (p. xiii), which as introductory sentence provides an excellent summary of what the book attempts. This is the lens through which the book reads ten or so narratives from the Hebrew Bible and the Apocrypha. The purpose of the book is to focus on "often unnoted acts of resistance" (p. xvii), especially acts which are "nonviolent in nature". Claassens engagement with the narratives is divided into four chapters in which we encounter resistance against "the violence of war" (Chapter 1), "the violence of rape" (Chapter 2), "the violence of heterarchy" (Chapter 3 ) and "the violence of precarity" (Chapter 4). In this review I will briefly sum up and critique her readings in these four chapters. Some are more convincing than others and my review will start by presenting a summary of each chapter as well as a discussion of weaknesses and strengths.

In Chapter 1 Claassens engages with two narratives, namely 2 Samuel 21, which is about "Rizpah's Lament", and 1 Samuel 25, which she titles as “Abigail's Hospitality". One objection against Claassens's reading of 1 Samuel 25 could be summed up as follows: "if you look for resistance in a text you will find it." Claassens's interpretation of the story of David, Abigail and Nabal is a case in point. The story is familiar. David is fleeing Saul and finds himself with his men in Carmel. David sends his young men to ask for food (or demand food) and Nabal turns down this request. Whereas some interpreters would describe David's actions as extortion reminiscent of modern-day gangers seeking "protection money," Claassens has a much more sympathetic view of David's request. For her David and his men are victims of hunger and Nabal fails to see this, whereas Abigail does. For Claassens Abigail performs two acts of resisting violence. The first one is providing food and the second is the words she speaks. In both cases Claassens masterly paints a very persuasive picture of how nourishing food and wise words could defuse a situation pregnant with violent potential.

Yet the main question is whether Abigail's actions exemplify resistance. Would it not be more apt to describe what Abigail does as a pragmatic act of survival? In both cases, feeding and speaking peaceful words, Claassens portrays Abigail as a woman who reminds us of "Woman Wisdom" of Proverbs 9. This interpretation is convincing, yet the result is the survival of Abigail and everybody who works with Nabal, with only Nabal not surviving. In defence of Claassens, she does conclude this section with a discussion on complexity (pp. 
29-30) in which she sums up some of the objections raised by feminists. Such as the fact that Abigail disappears from the text and never speaks again. She is just another trophy in David's trophy cabinet, but one could present a much more critical reading of the text. Nabal is a rich man from the tribe of Caleb and marrying his wife would provide an ideal stepping stone for the usurper to aim for bigger things. Abigail (like quite a few other women) is just another pawn in the larger chess game played by the men. In the last few chapters of the book Claassens often refers to the fact that "to survive is one of the ultimate forms of resistance" (p. 113). Abigail did indeed survive, but that was achieved by means of collaborating and it is not clear to me that this is indeed "resistance"?

Another aspect of Claassens's interpretation of the narratives is that she tends to shy away from exploring the "dark side" of Yahweh. This is particularly striking in the story of Rizpah. It is clear that Rizpah's silent lament is an act of resisting the violent world of men, but one culprit in this violent world who is never portrayed as such in Claassens's interpretation is Yahweh himself. It is presumably Yahweh who brings the famine and "diagnoses" the problem of blood guilt (v. 1), and it is God who relents in verse 14 in the last verse of the narrative. Also, the impalement committed by the Gibeonites is performed "before Yahweh", which is usually an expression for a sacrifice (v. 9). It seems that David is moved and indeed transformed by what Rizpah does, since verse 11 is clear that his hearing of the deeds of Rizpah leads to his actions of burying the bones of the sons of Saul. The narrative is not clear on what exactly triggers the transformation in Yahweh. Is it the lament of Rizpah, or the spilling of blood and thus the paying of the blood guilt? Verse 14 only says "after that" (NRSV), Yahweh relented, and it is not clear what the exact referent of "that" is. It seems that there is a missed opportunity in the book to engage with violent portrayals of Yahweh himself, or at least to contrast the portrayal of Yahweh in this story with the narratives Claassens discusses in Chapter 4, where the actions of Yahweh in response to the resistance from different women is explicitly presented.

In Chapter 2 Claassens interprets the stories of the rape of Tamar in 2 Samuel 13 and Susanna's prayer in the Apocrypha. The two narratives are aptly contrasted with each other, since they differ in one important regard and that is that Susanna successfully resisted being raped, whereas Tamar failed. The topic of resisting rape is sadly very relevant for the South African context and Claassens often draws from this context. About Tamar, Claassens stresses the role of lament (as with Rizpah) and the fact that "survival is the ultimate form of resistance" (I suppose as with Abigail). She concludes the discussion of Tamar with a plea to create "spaces for resistance" in which biblical stories like the one of Tamar could potentially play a role. Her discussion of Susanna should also be lauded, since books from the Apocrypha (as Protestants would refer to it) do no feature enough in academic studies of the Hebrew Bible. This section is also concluded with a discussion on how feminists are in two minds about what to 
make of Susanna. Claassens is more positive and would like to see the story as a counternarrative expressing resistance to rape. For Claassens it is important to also note the role that God plays in this narrative over against his lack of intervention in, for instance, the story of Jephthah (and I would add Rizpah). She is also not deterred by the fact that a young Daniel eventually saves Susanna and would rather highlight the fact that the saviour is young rather than focus on the fact that he is male.

Chapter 3 makes use of the term "heterarchy", coined by Carol Meyers, which understands that Israel is a far more complex society than generally believed, with different power structures where women could sometimes occupy positions of some power. The stories of the daughter of Jephthah in Judges 11 and the daughters of Zelophehad in Numbers 27 are analysed in this chapter. The story of Jephthah's daughter has been discussed by many and Claassens provides us with a fascinating representation of this debate, which also includes discussions of the Jewish reception of this story. At the beginning of the section Claassens acknowledges that "resistance is indeed limited but ... by no means insignificant" (p. 72) What is not mentioned, though, is the silence of God. Claassens did refer to this question posed by Phyllis Trible when she discussed Susanna (p. 63), but the question is never taken up again in her discussion of Judges 11. The discussion of Judges 11 is concluded with a discussion on the HIV and AIDS pandemic in Africa. It is not clear how the foolishness of "sacrificing a young woman" is somehow related to the "great folly of a widespread myth that having sex with a virgin daughter may cure one from HIV and Aids" (p. 82). Would this last section have not fitted better with the previous chapter, which dealt with rape?

The discussion of Numbers 27 and the efforts of the daughters of Zelophehad to keep their land by resisting patriarchal rules of inheritance also takes us to contemporary issues in the South African context. Claassens presents a well-balanced discussion in which she does not shy away from the complexity of the debate. Land is a matter of "human dignity", but in the larger narrative of the Pentateuch the Israelites are the conquerors and we are confronted with "competing claims of justice". Claassens fruitfully draws on insights from postcolonial studies using the narrative as a plea for seeing "the other" resonating with debates which are currently ongoing in South Africa.

The penultimate chapter of the book (Chapter 4) engages with stories which resist "precarity" and situates itself within a world where poverty could be described as "the worst form of violence". The chapter presents us with two basic discussions: one on the intertwined stories of Hagar and Sarah, and one on the stories of Ruth, Naomi and the other Tamar of Genesis 38. In the linked stories of Hagar and Sarah God responds clearly, first to the tears of Hagar and then to the laughter of Sarah. As mentioned before, it stills feels as if there is a missed opportunity here. Claassens could have explored the difference between 
the responsive God in these stories and the more reserved God we find in the stories of Rizpah and the daughter of Jephthah. Why does Yahweh respond to the resistance of some, but not so much to the resistance of others? Yet what Claassens shows clearly is that what all of the characters in Chapter 4 have in common is that they survive, and again the reader is reminded that to survive and especially to thrive is the ultimate form of resistance.

The book is concluded with the usual synthesising chapter, with the last two issues addressed being "complexity" and "hope"; this presents us with a healthy mix of honesty in facing reality and of hopefulness in changing it.

Prof Esias E. Meyer, Faculty of Theology and Religion, Room 2-41, Theology Building, University of Pretoria, Private Bag X20, Hatfield 0028, South Africa. DOI: https://doi.org/10.17159/2312-3621/2019/v32n1a15.

Christine Helmer, Steven L. McKenzie, Thomas Römer, Jens Schröter, Barry Dov Walfish, Eric Ziolkowski (eds.), Encyclopaedia of the Bible and its Reception 15: Kalam - Lectio Divina (Berlin, Boston: De Gruyter, 2017). Hardbound, xxix +1220 cols. ISBN 978-3-11-031332-1.

Volume 15 of the monumental Encyclopaedia of the Bible and Its Reception ( $E B R$; projected to contain 32 volumes in total) includes many entries on biblical books (1-2 Kings, Lamentations), persons, places/archaeological sites, words and people associated with the Bible and its reception which are of interest for students of the Ancient Near East, of Semitic Languages and of the Hebrew Bible/Old Testament.

The entry "King, Kingship" illustrates the EBR's approach to covering biblical persons, subjects, etc. and their variegated receptions in different contexts and genres. It consists of the following sub-entries:

A. Knapp, “King, Kingship I. Ancient Near East” (217-222), M. W. Hamilton, "King, Kingship II. Hebrew Bible/Old Testament" (222-224); H. Börm, "King, Kingship III. Greco-Roman Antiquity" (224-228); C. C. Caragounis, "King, Kingship IV. New Testament" (229-230); D. C. Flatto, "King, Kingship V. Judaism A. Second Temple, Hellenistic, and Rabbinic Judaism" (230-235); A. Shapira, "King, Kingship V. Judaism B. Medieval Judaism" (235-238); G. Seabourne, "King, Kingship V. Christianity" (238-240); S. V. Davidson, "King, Kingship VII. Other Religions" (240-241); M. Edwards, "King, Kingship VIII. Literature" (241-243); M. Patella, "King, Kingship IX. Visual Arts" (243-246); N. H. Petersen, "King, Kingship X. Music" (246-247) and S. Yang, "King, Kingship XI. Film” (247-249).

Other interesting entries in this volume are: C. A. Eberhart, "Kapporet" (26-30); E. A. Knauf, "Kedar" (96-98); M. Davis, "Kenyon, Kathleen Mary" 
(124-125); C.- L. Seow, "Keren-Happuch" (128-130); T. Wagner, "Keturah" (147-149); Y. Ofer, "Ketuvim (Writings)" (150-151), C.- L. Seow, "Keziah" (161-164); E. Gaß, "Kidnap, Kidnapping I. Hebrew Bible/Old Testament” (180183); R. C. Kashow, "Kidney I. Hebrew Bible/Old Testament” (187-189); K. H. Richards, "King James Version" (206-216); M. W. Hamilton, "Kingdom/Kingship of God I. Hebrew Bible/Old Testament" (253-255); A. M. Maeir, "Kingdoms of Israel and Judah I. History and Archaeology" ( 286-291); S. L. Adams, D. Moster, E. Morlok, J. Davis, "Kingdoms of Israel and Judah II. Judaism" (291-299); M. Page, "Kingdoms of Israel and Judah III. Film" (299301); T. Kauhanen, "Kingdoms, Books of" (301-303), "Kings (Books)" appears in eight subentries: S. L McKenzie (I. Hebrew Bible/Old Testament, 303-306), S. Moyise (II. New Testament, 306-309), R. L. Cohn (III. Judaism, 309-312), F. van Liere (IV. Christianity, 312-313), K. Killeen (V. Literature, 313-316), R. A. Leson (VI. Visual Arts, 316-319), S. Dowling Long (VII. Music, 319-321) and M. Page (VIII. Film, 321-322); T. M. Willis, "Kinship I. Hebrew Bible/Old Testament" (323-325); J. Rückl, "Kish (Person)" (345-351); R. K. Hawkins, "Kittim I. Hebrew Bible/Old Testament" (384-386); M. Bundvad, "Knowledge I. Ancient Near East and Hebrew Bible/Old Testament" (417-421); F. Ueberschaer, "Korah/Korahites I. Hebrew Bible/Old Testament" (455-456); H. Gzella, "Laban (Person) I. Hebrew Bible/Old Testament" (521-523); R. C. Kashow, "Labour (Childbirth) I. Hebrew Bible/Old Testament" (535-538); R. K. Hawkins, "Labour (Work) I. Hebrew Bible/Old Testament" (557-560); Y. Garfinkel, "Lachish" (590-594); M. Warner, "Lamb I. Hebrew Bible/Old Testament" (637-638); J. Schipper, "Lame, Lameness I. Hebrew Bible/Old Testament" (658-660); A. K. Schüle, "Lamech I. Hebrew Bible/Old Testament" (671-673); N. Samet, "Lament, Lamentation I. Ancient Near East and Hebrew Bible/Old Testament" (686-691); C. Frevel, "Lamentations, Book of I. Hebrew Bible/Old Testament" (736-741); E. Lapp, "Lamp, Lamps I. Archaeology" (768-774); M. Richelle, "Lamp, Lamps II. Ancient Near East and Hebrew Bible/Old Testament" (774-776); C. Levin, "Land I. Hebrew Bible/Old Testament" (781-784); R. K. Hawkins, "Laugh, Laughter I. Hebrew Bible/Old Testament" (933-935); S. Démare-Lafont, "Law I. Ancient Near East" (959963); A. C. Hagedorn, "Laugh, Laughter II. Hebrew Bible/Old Testament" (963967); Alphonso Groenewald, "Law, Book of the" (1025-1027; Groenewald of the University of Pretoria and Jonathan More of the George Whitefield College are the only two South African authors; More writes on "Kingdom/Kinship of God III. Judaism A. Second Temple and Hellenistic Judaism", 259-260); A. C. Hagedorn, "Lawgiver I. Hebrew Bible/Old Testament" (1027-1029); R. C. Kashow, "Lawless, Lawlessness" (1041-1044); M. A. Sweeney, "Lawsuit" (1064-1067); M. Sneed, "Lazy, Idle I. Ancient Near East" (1100-1102), M. Sneed, "Lazy, Idle II. Hebrew Bible/Old Testament" (1102-1103); F. Borchardt, "Leader, Leadership I. Hebrew Bible/Old Testament" (1121-1124); I. Fischer, "Leah I. Hebrew Bible/Old Testament" (1154-1158); S. Fischer, "Leaven I. Hebrew Bible/Old Testament" (1177-1179); C. McKinny, "Lebanon, Ancient I. 
Archaeology and History" (1191-1198) and J. Kamlah, "Lebanon, Ancient II. Hebrew Bible/Old Testament" (1198-1201).

As in previous volumes, this volume also covers the reception of the Bible in Europe and North America fairly well. The reception of the Bible in other parts of the world appears only rarely. While this may also be due to some of the methodological problems involved (see my survey "Recent Contributions to the Study of the Reception of the Bible and Their Implications for Biblical Studies in Africa", Religion and Theology 22, 2015, 329-383), in many cases it is simply a matter of ignorance, neglect and an exclusively Western perspective.

Exceptions in this volume are the entries by D. Daughrity on the "Kimbanguist Church" (2013-205) in the Congo and its reception of the Bible, and on "Lalibela" (626-629), a site of tremendous significance to Ethiopian Orthodox Christian churches, which have many connections to Judaism, as reflected in their theology, art and architecture (626). In the welcome entry, "Land IV. Christianity D. World Christianity" (786-798), J. K. Rutere from the Pan-African Christian University in Kenya includes references to the use and understanding of land by African clan systems in parallel to Josh 13-19, and the notion of ancestral lands. Here "World Christianity" means East Africa. Whether and how the reception of the Bible has had an impact or might have an impact on such parallels does not become clear. Several references to Africa appear in D. J. Fuller's entry, "Law V. Christianity F. World Christianity" (1016-1017).

While many of the entries on the reception of the Bible carefully trace this reception, other entries unfold their themes without much indication of which contents are to be understood as actual receptions of the Bible and how these have happened and are happening.

More than a quarter of a century after the publication of the substantial Anchor Bible Dictionary, it is welcome that the EBR offers an up-to-date, detailed, international dictionary of the Bible. Its attempt to cover the reception history of the Bible reflects recent trends in Biblical studies and cultural studies in general to include the reception history within their scope. This opens a wide field for new methodologies and research subjects.

Christoph Stenschke, Biblisch-Theologische Akademie Wiedenest and Department of Biblical and Ancient Studies University of South Africa, P O Box 392, Pretoria, 0003 Republic of South Africa. E-mail: Stenschke@wiedenest.de DOI: https://doi.org/10.17159/2312-3621/2019/v32n1a15. 
Meik Gerhards, Protevangelium. Zur Frage der kanonischen Geltung des Alten Testaments und seiner christologischen Auslegung, Stuttgarter Bibelstudien 237, Stuttgart: Katholisches Bibelwerk, 2017, 207 pages, softcover, $€$ 28.00, ISBN 978-3-460-03374-0

Das zu besprechende Buch widmet sich der äusserst aktuellen und zugleich oft stiefmütterlich behandelten Frage nach der christologischen Auslegung des Alten Testaments. Gleich zu Beginn des Vorworts betont der Verfasser, Privatdozent für Altes Testament in Rostock, dass es sich dabei um eine exegetische Frage handelt, die sich ganz besonders Alttestamentlern stellt, „da die Entscheidung, ob Jesus Christus als Mitte der ganzen Heiligen Schrift aufzuweisen ist, vor allem an alttestamentlichen Texten fällt" (S.7). Im weiteren Verlauf des Vorwortes hält Gerhards fest, dass der Graben in dieser Frage nicht entlang der Konfessionen verläuft, sondern durch die Konfessionen hindurch geht: „Von daher können die Überlegungen der vorliegenden Arbeit wohl in einer ökumenischen Gemeinschaft derer diskutiert werden, die der Überzeugung sind, dass es zu den Aufgaben der Exegese gehört, darauf hinzuarbeiten, dass aus der einen Heiligen Schrift Gottes Wort vernommen werden kann“"(S.11).

Das Buch hat zwei Hauptteile: Beim Ersten handelt es sich im Wesentlichen um, Überlegungen zur kanonischen Geltung des Alten Testaments und seiner christologischen Auslegung im Gespräch mit N. Slenczka“, angereichert durch vier Exkurse. Der zweite Hauptteil stellt exemplarisch die exegetische Frage nach der Berechtigung, in Gen 3 ein Protevangelium zu sehen.

In der Hinführung zum ersten Hauptteil befasst Gerhards sich mit dem Zueinander von historisch-kritischer Bibelwissenschaft und der traditionellen kirchlichen Schriftauslegung. Ein Zurück hinter die historisch-kritische Form der Bibelwissenschaft sei nicht möglich. Doch selbst wenn man die Schrift rein historisch-kritisch, ohne dogmatische Voreingenommenheit lese, werde man durch die biblischen Texte vor ein Entweder-Oder gestellt, den Anspruch Gottes anzuerkennen, oder zurückzuweisen. Daraus ergibt sich für Gerhards die Vorordnung der historisch-kritischen Auslegung vor die traditionelle Schriftauslegung: Zunächst sei der Text einer philologisch-literarischen Untersuchung im Rahmen historisch-kritischer Methodik auszulegen. Lässt man sich dann aber auf den historisch-kritisch ermittelten Sinn des Textes ein, so ermöglicht sich im Wagnis des Glaubens auch der Rückgriff auf ältere Auslegungstraditionen. Wenn Gerhards freilich schreibt, ohne historische Kritik lasse sich ,vielleicht kein einziger biblischer Text auch nur aus der Grundsprache ins Deutsche "übersetzen, stellt sich die Frage, ob er damit den Begriff der historischen Kritik nicht über Gebühr ausdehnt. Oder gab es vor dem Aufkommen der historischen Kritik etwa keine Bibelübersetzungen?

Die hermeneutischen Überlegungen geschehen in Auseinandersetzung mit N. Slenczka, der ja in den letzten Jahren dezidiert die Meinung vertreten hat, mit der historischen Kritik habe sich das Christuszeugnis des Alten Testaments 
als Illusion erwiesen, weshalb das Alte Testament zwar als Verstehenshintergrund für das Neue einen Wert behalte, aber nicht mehr Bestandteil des christlichen Kanons sein könne. Zunächst befasst sich Gerhards mit dem Verhältnis zwischen Judentum und Christentum (diese Überlegungen umfassen auch die ersten beiden Exkurse). Er widerspricht der Vorstellung Slenczkas, nach der sich das Christentum als eigenständige Religion aus dem Judentum heraus entwickelt und dabei den Sinn der Bibel Israels neu interpretiert habe. Vielmehr betont er mit L. Schwienhorst-Schönberger, dass weder Judentum noch Christentum direkter Adressat der Heiligen Schrift sei, sondern das Gottesvolk, in welchem es zu einer Auseinandersetzung über die rechte Interpretation kam, die zu einer Spaltung führte. Historisch gesehen hat die Kirche darum kein geringeres Recht am Alten Testament als das Judentum. Die Differenz der Auslegungen liegt letztlich in der Frage, „ob Jesus von Nazareth der in alttestamentlichen Texten im Voraus erwähnte Messias ist" (S.54). Bei allem Verständnis für das Anliegen, christlichem Antijudaismus entgegenzutreten, sei es aber nicht zielführend, die Auslegungsdifferenzen zwischen Judentum und Christentum einebnen zu wollen. Dieser Versuch ende immer in einer Relativierung des neutestamentlichen Christuszeugnisses. Doch die Notwendigkeit einer Nivellierung von Auslegungsdifferenzen könne aus dem Grundethos liberaler Gesellschaften gar nicht abgeleitet werden: " $\mathrm{Zu}$ diesem Ethos gehört [...], dass in einer Gesellschaft, die dem Einzelnen die freie Wahl eines Bekenntnisses zugesteht, religiöse Differenzen nicht nivelliert werden müssen“" (S.63).

Neben der Frage nach einem "historischen Recht" auf das Alte Testament sieht Gerhards auch in der entsubstantialisierten Christologie im Gefolge Schleiermachers einen Grund für Slenczkas Meinung, auf das Alte Testament verzichten zu können: "Wenn es nicht mehr darum geht, ob Jesus von Nazareth tatsächlich der Messias ist, von dem schon alttestamentliche Texte gesprochen haben, wenn es nur um religiöse oder soteriologische Erfahrungen geht, die sich auch heute noch als befreiende Deutung der eigenen Existenz bewähren, dann bedarf es des Alten Testaments als der heilsgeschichtlichen Vorstufe des Kommens Jesu nicht" (S.66). Während für Slenczka aus philosophischen Gründen eine Rückkehr in die transzendentale Unschuld einer substantiellen Christologie nur um den Preis der Unredlichkeit möglich ist, sei er aber jedenfalls im Blick auf das Neue Testament exegetisch in einer Position, die "auf Grund ihrer Prägung durch aufklärerische Dogmatik und wegen einer teilweise fragwürdigen Methodik äusserst kritisch zu bewerten“" sei (S.67). Gerhard selbst sieht sich auf dem Boden einer Theologie, die dem traditionellen ökumenischen Verständnis des Christentums verpflichtet ist und für die gilt, dass ein Bekenntnis zu Jesus als dem Christus ohne das Alte Testament nicht möglich ist.

Eine weitere Frage, mit der sich Gerhards im ersten Hauptteil befasst, ist die nach Heilsgeschichte und Geschichte. Während er in Exkurs III den in der Bibel gezeichneten heilsgeschichtlichen Bogen skizziert, der die Differenz von 
Altem und Neuem Testament überwölbt, stellt er in Exkurs IV die Frage nach dem Verhältnis von biblischer Geschichtsdarstellung und historisch-kritisch rekonstruierter Geschichte. Hier unterscheidet Gerhards zwischen Altem und Neuem Testament. Dass alttestamentliche Geschichtsschreibung weithin fiktiven Charakter habe, sei "heute nicht mehr zu bestreiten" (S.82). Es gehe im Alten Testament nicht um die Geschichte hinter dem Text, sondern um die Anamnese des Lesers vor dem Text. Dafür aber sei die biblische Darstellung das allein Wesentliche, die rekonstruierten historischen Hintergründe seien dagegen unwesentlich. Das gelte zunächst auch für das Neue Testament, doch sei die Rückfrage nach der Geschichte hinter dem Text da ungleich wichtiger, da die Texte an das Christusereignis heranführen. Nicht die Anamnese, sondern das Ereignis selbst ist hier die Hauptsache: "Ansonsten kommen wir zu der entkernten, entsubstantialisierten Form des Glaubens, der wohl kaum vermittelbar sein dürfte“ (S.85). Die von Slenczka suggerierte Alternative zwischen Tatsachenbericht und Ausdruck religiöser Empfindungen werde den Texten des Neuen Testaments insgesamt nicht gerecht. Hier möchte man doch fragen, ob diese Alternative denn den Texten des Alten Testaments gerecht wird? Zur geschichtlichen Frage gehört schliesslich auch Slenczkas Position, dass nur der ursprüngliche Sinn, d.h. die Autorintention Norm der Interpretation sein könne. Wie Gerhards zu Recht einwendet, ist diese Position aus hermeneutischer und literaturwissenschaftlicher Sicht so veraltet, "dass es überrascht, dass sie heute überhaupt noch in Betracht gezogen wird" (S.92). Ob ein Verständnis textgemäss sei, hänge nicht von der Autorintention $a b$, sondern davon, ob Sinnpotentiale im Text angelegt sind, selbst wenn sie dem Autor unbewusst gewesen sein mögen. Als Beispiel nennt er die Emmausgeschichte, in welcher der Auferstandene Christus den Emmausjüngern offenbar eine zu selektive Messiaserwartung durch ebenfalls im alttestamentlichen Text angelegte Sinnpotentiale korrigiere.

Im zweiten Hauptteil fragt Gerhards also nach einem Protevangelium in Gen 3, wobei er den Text zunächst nach historisch-kritischen Erkenntnissen philologisch-literarisch auslegen und erst in einem zweiten Schritt die alttestamentlichen Texte in ihrem ermittelten Sinngehalt mit dem neutestamentlichen Christusereignis korrelieren möchte.

Zunächst widmet sich Gerhards dem traditionell als Protevangelium verstandenen Vers Gen 3,15. Bei Luther benennt er drei Aspekte, die dazu führen, dass der Vers auf Jesus Christus hin gedeutet werden kann: Die Auffassung, dass die Schlange vom Satan als Instrument gebraucht wurde, die Auffassung, dass der Same der Frau auf ein Individuum ziele und die Auffassung, dass 3,15b einen endgültigen Sieg über die Schlange im Blick habe. Allen drei Aspekten widerspricht Gerhards mit seiner Textauslegung: Die Schlange sei weder mit dem Satan identisch, noch sei sie als dessen Werkzeug dargestellt, die Rede vom Samen der Frau ziele nicht auf ein Individuum, sondern auf die Menschheit insgesamt und im Blick sei nicht ein Endsieg über 
die Schlange, sondern eine relative Überlegenheit des Menschen über die Schlange. Damit sei Gen 3,15 nicht mit dem Sieg Christi über den Satan zu korrelieren und nicht als Protevangelium zu bezeichnen. Leider äussert sich Gerhards nicht zur Berechtigung des Neuen Testaments selbst, diesen Bezug herzustellen (einerseits in der Versuchungsgeschichte Jesu mit ihrem Bezug auf Ps 91,11-13, andererseits in Offb 12). Nicht alle Argumente Gerhards überzeugen gleichermassen. Es ist $\mathrm{ja}$ an verschiedenen Orten im Neuen Testament gerade eine Pointe der Christologie, dass Christus als Individuum ein Kollektiv repräsentiert. Dürfen kollektiv gefasste Texte nicht auf Christus bezogen werden, dann fallen u.a. auch die Nachkommensverheissung an Abraham (Gal 3,16) und die vielfältigen Gottesknechtsbezüge unter dasselbe Verdikt.

Verwirft Gerhards die Deutung von Gen 3,15 als Protevangelium, so liefert er aber gleich einen neuen Vorschlag für ein Protevangelium in Gen 3, nämlich V.21. Im Rahmen der Menschwerdungserzählung Gen 2f. ist das Bewusstsein der Nacktheit mit der Erkenntnis von Gut und Böse verbunden. Während in Gen 3,7 sich die Menschen selber Kleider herstellen, um sich voneinander abzugrenzen, haben die Fellröcke in 3,21, die Gott den Menschen macht, eine Schutzfunktion. Die Bekleidung des Menschen durch Gott bringt zum Ausdruck, dass der Mensch in der wilden Natur nicht auf sich alleine gestellt ist, sondern dass Gott dem Menschen nach dem Sündenfall eine neue, nicht von der Sünde bestimmte, Existenzweise schenkt. Diese Existenzweise wird nach Gerhards in Gen 3,21 allerdings nur als Perspektive eingeführt und nicht eingelöst. Verwirklicht wird sie im Rahmen der Urgeschichte und auch der gesamten Genesis nicht. Die Erwartung dieser neuen Existenzweise lebt in der prophetischen Erwartung des Alten Testaments weiter, ohne aber zur Erfüllung zu kommen. Entscheidend ist nun für Gerhards, dass die Einsicht, dass Gott schon nach dem Sündenfall an einer Klärung des durch die Sünde getrübten Zustandes gelegen ist, allein aus Gen 3,21 zu gewinnen ist, ohne Berücksichtigung des neutestamentlichen Christuszeugnisses: "Wenn aber an dieser Stelle innerhalb der erzählten Welt von Gen 2f. kein leeres Bild erscheint, muss es eine Erfüllung geben: Diese findet der Leser, der sich auf die Einheit der Heiligen Schrift einlässt, in der Erlösung durch Jesus Christus“" (S. 163). Eine Vertiefung findet die christologische Deutung von Gen 3,21 dann in der Bekleidungsmetaphorik insbesondere bei Paulus, aber auch schon innerhalb des Alten Testament im Tieropfer, das selber als Typos auf den Tod Christi hinweist.

An den Beispielen Gen 3,15 und Gen 3,21 verdeutlicht Gerhards somit sein methodisches Vorgehen für eine christologische Auslegung des Alten Testaments. Der alttestamentliche Text muss in seinem Sinngehalt aus sich selbst heraus ausgelegt werden. Wenn der herausgearbeitete Sinngehalt dann in Korrelation mit dem Christusereignis zu bringen ist, ist eine christologische Deutung in einem zweiten Auslegungsdurchgang legitim. 
Gerhards legt mit dieser Studie ein mutiges und notwendiges Buch vor. Wenn er im Vorwort bezweifelt, ob es von Bibelwissenschaftlern überhaupt zur Kenntnis genommen wird (S.11), spricht er ein wahres Wort aus, hat sich doch der Hauptstrom der Bibelwissenschaften von solchen theologischen Fragestellungen weitgehend verabschiedet und sich auf das Gebiet der Religionsgeschichte zurückgezogen. Doch wenn die Kirche das Alte Testament nicht verlieren will und wenn die Bibelwissenschaften das Gespräch mit der Kirche nicht abbrechen wollen, so wird es nötig sein, ganz neu zu fragen, was eine christliche Auslegung des Alten Testaments sein kann und soll. Dabei sollte auch die Herablassung, mit der die kritische Bibelwissenschaft gelegentlich auf die angeblich naive und "vor-kritische" traditionelle Auslegung herabschaut, abgelegt werden. In Vielem kann ich Gerhards folgen, gerade im methodischen Zweischritt, den alttestamentlichen Text zunächst aus sich selbst heraus zu verstehen, um dann vom Christusereignis her nochmals zurückzukehren und zu sehen, was das Licht dieses Ereignisses zutage fördert, das zunächst nicht klar sichtbar war. Allerdings geht Gerhards m.E. in seinen Überlegungen nicht immer weit genug. Drei Punkte seien herausgegriffen:

Verschiedentlich spricht Gerhards über die historische Kritik in einer Art und Weise, die den Anschein erweckt, als ob es sich bei ihr im Unterschied zur traditionellen kirchlichen Exegese um eine neutrale Herangehensweise an die Texte handle, welche objektive Resultate sicherstelle. Hier würde ich mir wünschen, dass Gerhards auch die ideologischen Prämissen einer von der Aufklärung her kommenden Bibelwissenschaft stärker reflektieren würde. Es gehört natürlich zu den Topoi der Aufklärung, dass man allen anderen ihren Standpunkt vorhält, selbst aber keinen zu haben vorgibt, sondern angeblich „,interesselos“ und „objektiv“ an die Sache herangeht. Doch dass dies eine Illusion ist, sollte nach Gadamer selbstverständlich sein. Auch die historische Kritik muss methodisch und ideologisch kritisierbar bleiben.

Das Verhältnis von biblisch dargestellter Heilsgeschichte und historischkritisch rekonstruierter Geschichte ist nicht zufriedenstellend geklärt und bedarf differenzierterer Reflexion. Die Aussage, es sei ,heute“ nicht mehr zu bestreiten, dass alttestamentliche Geschichtsschreibung weithin Literatur von fiktionalem Charakter sei, ist nicht nur als Totschlagargument formuliert (wer diese Behauptung bestreitet, ist offenbar kein "Heutiger"), sondern auch sachlich fragwürdig (Anamnese bezieht sich nicht auf Fiktion) und theologisch wenig reflektiert. Wie M. Sternberg in seinem Buch The Poetics of Biblical Narrative schreibt (in deutscher Übersetzung zitiert nach B.S. Childs, Theologie der einen Bibel, Bd. 1, 1994, 40): "Wenn die biblischen Erzählungen als Fiktionen geschrieben wurden und als solche zu lesen wären, dann würde sich Gott vom Herrn der Geschichte zu einem Geschöpf der Einbildungskraft verwandeln, mit allen zerstörerischen Folgen“. 
So sehr ich das Plädoyer gegen die entsubstantialisierte Christologie Schleiermachers und Slenczkas unterstütze: Gerade zur Substanz neutestamentlicher Christologie gehört die Präexistenz Christi, die auch für die Auslegung des Alten Testaments im Neuen eine zentrale Rolle spielt: Das Alte Testament zeugt von Christus, weil Christus im alttestamentlichen Gottesvolk anwesend war. Das Christuszeugnis des Alten Testaments wird aber nur durch das Christusereignis, das offenbart, was zuvor verborgen war, enthüllt. Das führt im Neuen Testament zu einer Art Relecture des Alten Testaments, allerdings verbunden mit dem Anspruch, dass das, was neu entdeckt wird, bereits in den Texten selbst angelegt ist. Der Sache nach kann ich dann Gerhards durchaus folgen, dass man also zuerst die alttestamentlichen Texte aus sich selbst heraus auslegen kann, bevor man vom Christusereignis zu ihnen zurückkehrt. Doch wäre es meines Erachtens unerlässlich für die Frage nach einer christologischen Auslegung des Alten Testaments, zu bedenken, was für hermeneutische Schlussfolgerungen aus der neutestamentlichen Christologie konkret zu ziehen sind. Auf jeden Fall darf christologische Auslegung dann nicht nur heissen, dass Christus im Alten Testament als der Abwesende angekündigt wird, sondern auch, dass er im Alten Testament selbst verborgen anwesend ist.

Ungeachtet der genannten Kritikpunkte ist Meik Gerhards dafür zu danken, dass er dieses heisse Eisen angefasst hat, was sicher nicht nur Applaus einbringen wird. Es enthält viele weiterführende Anregungen zur Frage nach der kanonischen Geltung des Alten Testaments und seiner christologischen Auslegungen und verdient Beachtung.

Benjamin Kilchör, Staatsunabhängige Theologische Hochschule Basel (Switzerland) and Dept. of Ancient Languages at the University of Pretoria (South Africa), Grüenaustrasse 21, CH-8624 Grüt (Switzerland). Email: benjamin.kilchoer@sthbasel.ch; DOI: https://doi.org/10.17159/2312-3621/2019 $\underline{\text { lv32n1a15. }}$

Christiane Wüste. Fels - Geier - Eltern: Untersuchungen zum Gottesbild des Moseliedes (Dtn 32). Bonner Biblische Beiträge 182. Göttingen: V\&R Unipress / Bonn University Press, 2018. 281 Seiten. Leinen. EUR 50. ISBN 978-3-8471-0643-2

Die Monographie stellt eine Doktordissertation der Katholisch-Theologischen Fakultät der Universität Bonn (Doktorvater: Ulrich Berges) dar. Die Verfasserin (Vfn.) widmet sich einem gleicherweise schwierigen wie einflussreichen poetischen Text, dem sogenannten Moselied in Dtn 32, 1-43. Dabei richtet sich ihr Augenmerk insbesondere auf dessen Gottesbild mit den drei im Titel enannten Bildbereichen. 
In ihrer Einleitung geht Vfn. von der Problematik „Gewalt im Gottesbild““ aus und wählt mit Dtn 32 einen Text, in dem diese Thematik zur Sprache kommt (auch wenn das Moselied nicht zu den "klassischen" Gewalttexten zählt). Sie geht aber nicht von den eigentlichen Gewaltaussagen aus, sondern wählt drei Gottesmetaphern, die "auf den ersten Blick positiv konnotiert sind und so den gewalthaltigen Seiten JHWHs im Lied gegenüberstehen“ (16). Auf diese Weise soll ein zutreffenderes, zwischen polaren Aussagen oszillierendes Gottesverständnis wahrgenommen werden. Auf die Theoriediskussion zu Metaphern geht sie nicht ein, erwähnt aber kurz ihre Orientierung am Interaktionsverständnis von P. Ricœur.

Im ersten Hauptkapitel ("Grundlegungen") erfolgt die Texterfassung in synchroner und diachroner Weise. Das poetische Stück wird in Nebeneinanderstellung von hebräischem Text (MT; mit kolometrischer, aber nicht strophischer Strukturierung) und deutscher Übersetzung dargeboten. Es folgen Hinweise zu Textkritik und Grammatik (ausführlich werden V. 8 und v.a. V. 43 erörtert; Q- und LXX-Fassungen seien älter als MT). Was die Makrostruktur betrifft, geht Vfn. von drei Hauptteilen aus (Geschichtsrückblick: 8-14.1518 | erste Gottesede: 20-25.26-35 | zweite Gottesrede: 37-38.39-42). Diese sind durch "Brückenverse" (19|36) verbunden und werden durch Proömium und Schlussvers (1-7 | 43) gerahmt. Ergänzend dazu wird umsichtig die komplexe Kommunikations- und Zeitstruktur erfasst. Hinsichtlich der Entstehungsgeschichte ist gemäss der Vfn. der älteste Kern (V. 8-18.19-25) und eine erste Erweiterung (V. 26-35) vorexilisch (denkbar); Proömium, Schluss (V. 36-43) und Lied insgesamt sind nachexilisch. Dabei ist ein Schwanken insofern zu konstatieren, als sie der Auffassung von E. Otto zuneigt (angesichts hoher Intertextualität eine Art Summarium und einer der jüngsten Texte des ATs), aber gleichwohl von vorexilischen Vorstufen ausgeht, die literarisch freilich nicht greifbar seien. Gattungsmässig wird das Moselied als Mischung aus Lehre, Ermahnung, Geschichte und theologischer Reflexion eingestuft, das Identität und Orientierung bietet.

Der Hauptteil (107-247) bietet die eingehende Erarbeitung der drei Gottesmetaphern. Der "Fels" erscheint vielfach (V. 4.15.18.30.31.37, in V. 31 auch im Blick auf Fremdgötter), erweist sich als Leitbegriff des Lieds und als gewichtigstes der drei Bildmotive. Im Blick auf die kanonische Abfolge nimmt dieses Gottesepitheton hier seinen Anfang; weitere Verwendungsschwerpunkte liegen in den Büchern Samuel (poetischer Rahmen) und Psalter (David-Psalter I + II, beginnend mit Ps $18=2$. Sam 22). Ausgehend von der realen Schutzsuche vor Feinden auf Fluchtfelsen (Felsklüfte, -spalten wären in den Vorstellungshorizont miteinzubeziehen) steht das Epitheton für von Gott gewährte Zuflucht, Schutz und Rettung. Dabei sieht Vfn. in Dtn 32 den Endpunkt einer Begriffsentwicklung (De-Metaphorisierung, Lexikalisierung) - eine Einschätzung, die von diskutablen Parametern (u.a. Ps 81 älter als Dtn 32) abhängt. Die einzelnen Belege werden exegetisch minutiös durchgearbeitet (auch 
bei den andern beiden Metaphern). Die Rede von JHWH als "Geier" (gegenüber "Adler" von Vfn. aufgrund ikonographischer Quellen präferiert) beschränkt sich auf V. 11 (vgl. Ex 19,4). Mit dem Motiv vom "Tragen" auf Flügeln verbindet sich nuancenreich Schutz, Begleitung und Stärke. Zu denken ist an das Kreisen des Muttervogels über seinen Jungen.- Zu „JHWH als Eltern“ (die Bezeichnung ist nicht unproblematisch, weil so nie verwendet) behandelt Vfn. V. 5-6 sowie 18-20. Von Gott als "Vater" wird mit Blick auf den David-König (3 mal in Chr, 2. Sam 7,14; Ps 89,27, dazu Ps 2,7: j-l-d wird nicht mit "zeugen", sondern "gebären" übersetzt) und Israel (neben V. 6 Prophetenbelege und 1. Chr 29,10) gesprochen. Von ihm als "Mutter" ist in der Bibel nie direkt, sondern über "mütterliche" Verbalaussagen die Rede. In V. 6 wird der "Vater" als "(zeugender) Hervorbringer" erwähnt. In V. 18 ist die b-Zeile weiblich konnotiert (,unter Wehen hervorbringen"); die Aussage der a-Zeile lässt sich "männlich" (j-l-d,,zeugen") und damit komplementär oder ebenfalls "weiblich“ (j-l-d "gebären") verstehen. Vfn. entscheidet sich für die zweite Lesart und sagt, dass sich aus Dtn 32 ein "zweigeschlechtliches" Bild ergebe: JHWH werde in seinem Verhalten Israel gegenüber mit Vater und Mutter verglichen. Ergänzend dazu wird das Verhalten Israels als "Kinder" (V. 5-6.19.20) samt den daraus resultierenden Folgen charakterisiert.

Im Schlusskapitel werden die drei Gottesmetaphern im gesamten Moselied bedacht: hinsichtlich ihrer Funktionen, Bedeutungen und Interaktionen, dazu im Blick auf Sinnanreicherungen durch intertextuelle Verbindungen. Am Ende kehrt die Vfn. zur Ausgangsproblematik der göttlichen Gewalt (im Moselied) zurück und bilanziert: "Die 'positiven' und 'negativen" Seiten [JHWHs] können nicht gegeneinander ausgespielt werden ... Die positiven Konnotationen [der Gottesmetaphern] überwiegen deutlich: JHWH ist als Fels, Geier und Eltern zunächst der gerechte, lebensspendende, schützende, tragende und zugewandte Gott, der jedoch in dem Fall, dass seine Einzigkeit von Israel oder den Feinden nicht anerkannt wird, mit strafendem Gerichtshandeln reagiert ... Nur in diesem Ineinsfallen der Gegensätze, in dieser Komplexität ist vom biblischen Gott zu sprechen" (257). Abkürzungs- und Literaturverzeichnis runden den Band ab; Register fehlen.

Angesichts des Ausgangspunkts bei der Gewaltfrage sind die diesbezüglichen Erörterung am Schluss (zu) knapp. Insgesamt aber zeichnet sich die Monographie durch solide Arbeit in exegetisch-theologischer Hinsicht mit sorgfältig herausgearbeitetem Ertrag aus. Methodisch ergiebig liegt der Schwerpunkt auf begriffs-, motiv- und traditionsgeschichtlichen Untersuchungen. Die Ausarbeitung der drei Gottesbilder ist gewinnbringend und anschlussfähig für weitere Studien zu diesem bedeutenden Abschlusstext im Deuteronomium (und Pentateuch). Ob dieser auch (literar)geschichtlich - wie die Vfn. (mit E. Otto) meint - ein Abschlusstext oder doch eher ein Ausgangstext für die kanonisch nachfolgenden Texte ist, bleibt die Frage (Zwischenversionen scheinen weniger wahrscheinlich). Aufgrund des Mangels an sicher zu 
datierenden "Ankertexten" verlaufen die Diskussionen schnell zirkulär. Die Monographie ist davon nicht unberührt, ihr Ertrag ist aber unabhängig der Annahmen zu Datierung und Diachronie (Einheitlichkeit) gegeben.

Beat Weber, Research Associate of the Department of Ancient and Modern Languages and Cultures, University of Pretoria, Pretoria, South Africa. E-mail: weber-lehnherr@sunrise.ch. DOI: https://doi.org/10.17159/2312-3621/2019/v3 $\underline{2 n 1 a 15 .}$ 